\title{
Hidden blood loss and its influencing factors after minimally invasive percutaneous transpedicular screw fixation in thoracolumbar fracture
}

\section{Xin Yue}

First Affiliated Hospital of Dalian Medical University

\section{Kaige Mao}

Dalian Medical University

\section{Ming Yang}

First Affiliated Hospital of Dalian Medical University

Wentao Zhang

First Affiliated Hospital of Dalian Medical University

Zhonghai Li ( $\square$ lizhonghaispine@126.com )

First Affiliated Hospital of Dalian Medical University https://orcid.org/0000-0003-4735-1193

\section{Research article}

Keywords: Hidden blood loss (HBL), Risk factors, Minimally invasive percutaneous transpedicular screw fixation (MIPTSF), Multiple regression analysis, Complication

Posted Date: April 28th, 2021

DOl: https://doi.org/10.21203/rs.3.rs-445719/v1

License: (1) This work is licensed under a Creative Commons Attribution 4.0 International License. Read Full License 


\section{Abstract}

Objective: This study aimed to investigate the amount of hidden blood loss (HBL) and its influencing factors after minimally invasive percutaneous transpedicular screw fixation (MIPTSF) in thoracolumbar fracture.

Summary of Background Data: MIPTSF is generally accepted as a minimally invasive treatment for thoracolumbar fracture. However, HBL caused by this procedure is usually disregarded.

Materials and Methods: Between October 2017 and December 2020, a total of 146 patients (106 males and 40 females, age range 21-59 years) were retrospectively examined, and their clinical and radiological data were recorded and analyzed. The Pearson or Spearman correlation analysis was used to investigate an association between patient's characteristics and HBL. Multivariate linear regression analysis was performed to elucidate the related clinical or radiological factors of HBL.

Results: A substantial amount of HBL (164.00 $\pm 112.02 \mathrm{ml}, 40.65 \%$ of TBL) occurred after transpedicular screw internal fixation. Multivariate linear regression analysis revealed that $\mathrm{HBL}$ was positively associated with total blood loss $(T B L)(P=.000)$, percentage of vertebral height loss $(V H L)(P=.000)$, percentage of vertebral height restoration (VHR) $(P=.000)$, numbers of fractured vertebrae $(P=.013)$, and numbers of fixed vertebral segments $(P=.002)$.

Conclusion: A large amount of HBL was incurred in patients undergoing MIPTSF in thoracolumbar fracture. More importantly, TBL, percentage of VHL, percentage of VHR, the numbers of fractured vertebrae and fixed vertebral segments were independent risk factors for HBL.

\section{Background}

The thoracolumbar spine is one of the area's most commonly affected by spinal fractures ${ }^{1,2}$. However, conventional open posterior pedicle screw fixation causes increased intraoperative bleeding, a higher infection rate, postoperative back pain, delayed functional rehabilitation, and aggravation of posterior ligamentous complex (PLC) injury ${ }^{3-5}$. With advances in surgical techniques and instrumentation, the percutaneous approach has been successfully applied for pedicle screw fixation to treat thoracolumbar fracture ${ }^{6}$. The percutaneous approach allows spine surgeons to insert pedicle screws and rods and to connect them percutaneously through small skin incisions. Moreover, this system avoids the disadvantages of conventional surgical treatment, minimizes soft tissue injury, reduces intraoperative blood loss, and results in better postoperative pain scores than other approaches ${ }^{7,8}$. According to past clinical experience, MIPTSF is associated with a relatively low perioperative blood loss because of small incision, reduced muscular dissection, and short operative time ${ }^{9}$. But, the patients with thoracolumbar fractures tend to have a lower postoperative HB level than anticipated after surgery despite the apparently satisfactory perioperative management of blood loss. Previous studies examined only the volume of 
visible blood loss in the perioperative period. However, hidden blood loss (HBL) penetrating tissues, retained in a dead space, and lost due to hemolysis is often disregarded by orthopedic surgeons ${ }^{10}$.

Hidden blood loss (HBL) is not usually recognized by general assessment because of its invisibility ${ }^{11}$. HBL may exacerbate postoperative hemoglobin drop, affect postoperative outcomes, such as medical complications, increased blood transfusion risks, and prolonged postoperative rehabilitation ${ }^{12}$. The issue of HBL has been noted in other fields of orthopedic surgery. The concept of HBL was first put forward by Sehat in $2000^{11}$. Sehat et al. reported that the proportion of HBL was $50 \%$ of the TBL in total knee arthroplasty. Xu et al reported the mean hidden loss calculated with our recommendable method was $362.8 \mathrm{ml}$ and $47 \%$ of total loss in lumbar fusion surgery ${ }^{13}$. However, few studies have considered $\mathrm{HBL}$ in MIPTSF surgery during treatment of AO type A1-A3 thoracolumbar fractures with no neurological symptoms. Therefore, we retrospectively reviewed medical data of patients who underwent MIPTSF in our department in an attempt to evaluate HBL and identified the influencing factors of HBL.

\section{Methods}

\section{Patient Population}

This was a retrospective clinical study. The review of clinical database between October 2017 and December 2020 at one single center (First Affiliated Hospital of Dalian Medical University) was conducted. The study population included 146 patients aged 18 years or older who had AO type A1-A3 thoracolumbar fractures with radiographic evidence and hadn't symptoms of nervous system damage treated by MIPTSF alone. Patient's data were collected from the electronic medical records system of our institution. The information gathered including gender, age, height, weight, body mass index (BMI), hypertension (i.e., blood pressure $\geq 140 / 90 \mathrm{mmHg}$ ), diabetes mellitus (i.e., fasting blood-glucose $\geq 6.1$ $\mathrm{mmol} / \mathrm{L}$ ), smoking, drinking, using hormones, combining with other fractures, low immunity, surgical duration, hospital stay, muscle thickness, subcutaneous fat thickness, muscle thickness/subcutaneous fat thickness, fracture classification, numbers of fracture segments, numbers of fixed vertebral segments preoperative and postoperative hematocrit (HCT and hemoglobin (HB), prothrombin time (PT), activated partial thromboplastin time (APTT), thrombin time (TT), fibrinogen, and platelet (PLT), percentage of vertebral height loss, percentage of vertebral height restoration. Preoperative magnetic resonance imaging (MRI) was used to determine the distance of the lamina from the skin surface, thickness of the paraspinal muscles, and thickness of the subcutaneous fat. These measurements were all performed at the level of L1 using sagittal views (Fig. 1). And pre-, intra-, postoperative findings were recorded as well. All of the operations were performed by only one experienced surgeon.

\section{Inclusion and exclusion criteria}

The inclusion criteria for the study were: (1) age of $18 \sim 60$ years old, no gender preference, (2) A1-A3 thoracolumbar fractures (T11-L3); (3) surgical method: minimally invasive percutaneous transpedicular screw fixation. Our exclusion criteria were old thoracolumbar fractures, spine infection, spinal cord 
compression syndrome, severe cardiopulmonary comorbidity, major coagulopathy, and patients with symptoms of nervous system damage, liver cirrhosis or uremia.

\section{Management of blood loss}

No patient received blood transfusion throughout the assessment period. All of the patients underwent a full blood count, including HCT, and HB before the surgery and 2 or 3 days after the surgery for calculation of blood loss. No drainage was typically placed in any of the patients. There was little visible blood loss after surgery, therefore, postoperative blood loss could be ignored.

\section{Calculation of hidden blood loss}

Firstly, patient's blood volume (PBV) was estimated in accordance with the formula of Nadler et $\mathrm{al}^{14}$. PBV $(L)=k 1 \times$ height $(m) 3+k 2 \times$ weight $(\mathrm{kg}) 2+\mathrm{k} 3$; where $\mathrm{k} 1=0.3669, \mathrm{k} 2=0.03219$ and $\mathrm{k} 3=0.6041$ for males, and $\mathrm{k} 1$ for females. $=0.3561, \mathrm{k} 2=0.03308$ and $\mathrm{k} 3=0.1833$.

Secondly, according to the method of Gross et al. ${ }^{15}$, the TBL was calculated based on the HCT level and the PBV, as follows: TBL $(\mathrm{mL})=\mathrm{PBV}(\mathrm{L}) \times(\mathrm{HCT}$ pre $-\mathrm{HCTpost}) / \mathrm{HCT}$ ave, where HCTpre is the initial preoperative HCT, HCTpost is the HCT on the second or third day postoperatively, and HCTave is the average of the Hctpre and the Hctpost.

Finally, the method of Sehat et al. was used to calculate the HBL, as follows: $H B L(m L)=T B L(m L)-V B L$ $(\mathrm{mL})^{11}$. Since no drainage was typically placed in any of the patients, intraoperative blood loss was equal to VBL, VBL was given by the measured suction loss and blood loss in swabs, and recorded by the anesthetists.

\section{The definition of anemia}

According to the World Health Organization, anemia is characterized by HB levels of $<120 \mathrm{~g} / \mathrm{L}$ for women and $<130 \mathrm{~g} / \mathrm{L}$ for men) ${ }^{16}$.

\section{Calculation of the percentage of vertebral height loss and restoration}

All of the included cases were examined using plain radiographs. The predicted height of each fractured vertebra was calculated according to the average height of the two adjacent vertebrae. And the anterior vertebral height loss and restoration was measured according to the affected vertebral body. The percentages of vertebral height loss (VHL) and vertebral height restoration (VHR) were calculated with the following equations ${ }^{17}$ :

VBHave $=(\mathrm{VBHa}+\mathrm{VBHb}) / 2$

VHL $(\%)=($ VBHave - VBHpre $) /$ VBHave $\times 100 \%$,

VHR $(\%)=($ VBHpost - VBHpre) / VBHave $\times 100 \%$, 
where VBHave is the average height of the 2 adjacent vertebrae, and VBHpre is the preoperative anterior vertebral body height, and VBHpost is the postoperative anterior vertebral body height. (Fig. 2)

\section{Statistical analysis}

All of the independent variables were incorporated into the model using the method of "Enter." Data analyses were performed with the SPSS 23.0 software. (International Business Machines Corporation, Armonk, NY). A chi-squared test was adopted to compare the preoperative and postoperative incidence of anemia. Pearson's correlation (used for the normal data), Spearman's correlation analysis (used for the non-normal data), and multivariate linear regression analysis were performed to evaluate the influencing factors associated with HBL. In all analyses, $P<0.05$ was taken to indicate statistical significance.

\section{Results}

A total of 146 patients were reviewed retrospectively in this study. Among these patients were 106 males and 40 females, with a mean age of 42.31 (range 21-59) years. Table 1 summarizes the demographic and clinical characteristics. The mean muscle thickness was $31.61 \pm 7.84 \mathrm{~mm}$, while the mean subcutaneous fat thickness was $19.84 \pm 6.19 \mathrm{~mm}$. The mean preoperative HCT and HB were $38.23 \pm 4.41 \%$ and $124.86 \pm 14.36 \mathrm{~g} / \mathrm{l}$. The mean postoperative HCT and HB were $35.18 \pm 4.51 \%$ and $103.92 \pm 13.67 \mathrm{~g} / \mathrm{l}$. The mean PBV was $4.87 \pm 0.71 \mathrm{~L}$. The mean HBL was $164.00 \pm 112.02 \mathrm{ml}, 40.65 \%$ of $\mathrm{TBL}$, indicating a considerable amount of $\mathrm{HBL}$, which was much higher than we had expected. The mean VBL was $239.45 \pm 130.17 \mathrm{ml}$. The mean TBL was $403.45 \pm 182.25 \mathrm{ml}$. 76 patients suffered from preoperative anemia, and 56 patients developed anemia after surgery (Fig. 3). There were significant differences between preand postoperative HCT $(P<0.001)$ and HB $(P<0.001)$ (Table 2).

The Pearson or Spearman correlation analysis for HBL found the following parameters with a $\mathrm{P}<0.05$ (Table 3): TBL $(P=0.000), B M I(P=0.000)$, muscle thickness $(P=0.000)$, subcutaneous fat thickness $(P$ $=0.000)$, surgical duration $(P=0.000), P T(P=0.000)$, APTT $(P=0.000)$, TT $(P=0.038)$, diabetes mellitus $(P=.048)$, fracture classification (type $A 1-A 3)(P=.000)$, percentage of vertebral height loss $(P=.000)$, percentage of vertebral height restoration $(P=.000)$, numbers of fractured vertebrae $(P=.000)$, and numbers of fixed vertebral segments $(P=.000)$.

Next, we performed multiple and stepwise linear regression analysis to explore the association between $H B L$ and the influential factors mentioned earlier. The TBL $(P=.000)$, percentage of vertebral height loss $(P=.000)$, percentage of vertebral height restoration $(P=.000)$, numbers of fractured vertebrae $(P=.013)$, and numbers of fixed vertebral segments $(P=.002)$ were independent risk factors for $\mathrm{HBL}$ (Table 4). The results indicated that other factors were not significantly correlated with $\mathrm{HBL}$.

\section{Discussion}


Studies on HBL after orthopedic surgery have mostly focused on total hip arthroplasty (THA), total knee arthroplasty (TKA), and ALIF/PLIF surgery ${ }^{18}$. In a work on anterior/posterior lumbar fusion surgery (ALIF/PLIF), HBL was approximately $40 \%$ of TBL ${ }^{12,18}$. Chen et al. ${ }^{19}$ reviewed and analyzed of the patients undergoing conventional posterior open approach, the average $\mathrm{HBL}$ was $382 \pm 153.8 \mathrm{~mL}$; and the average $\mathrm{HBL}$ of patients undergoing percutaneous approach was $240.0 \pm 65.1 \mathrm{~mL}$. In our study, a substantial amount of HBL $(164.00 \pm 112.02 \mathrm{ml}, 40.65 \%$ of TBL) occurred after MIPTSF., the obtained amount was much greater than that of visible intraoperative blood loss. Some studies suggest that for patients undergoing total hip replacement, $\mathrm{HBL}$ is positively correlated with changes in BMI, blood transfusion, incision length, preoperative and postoperative $\mathrm{HCT}$, and negatively correlated with age ${ }^{20}$. Nevertheless, there have been no previous studies regarding the influential factors correlated to the HBL during the MIPTSF of AO type A1-A3 thoracolumbar fractures. In this study, we investigated and identified the risk factors of HBL following this surgery by multivariate linear regression analysis. The results proposed that the TBL, percentage of vertebral height loss, percentage of vertebral height restoration, numbers of fractured vertebrae, and numbers of fixed vertebral segments were positive independent risk factors for HBL.

Our statistical analysis showed that the patients who had massive TBL suffered from more HBL than those who have little TBL. TBL was the independent risk factor, which may have to do with PBV, because TBL is calculated by multiplying PBV by changes of HCT and subtracting the IBL according to the Gross formula ${ }^{15}$, which might relate to the patient's weight and height. However, BMI had not been identified as a risk factor in our study, although body mass index was also calculated by weight and height. Based on collected data in our study, it was easy to find that HBL is directly related to a large amount of blood loss.

HBL during orthopedic surgery is generally accepted as being due to blood infiltration into tissue compartments and loss due to hemolysis ${ }^{21,22}$. Our study found that the percentage of vertebral height loss and the percentage of vertebral height restoration were correlated with HBL. Vertebra involves cancellous bone, and its blood supply is abundant. The expansion of vertebral cavity will cause internal bleeding. The recovery of fractured vertebral body height may lead to enlarged cavity, and the space around vertebral body may be enlarged. We suspect that the blood would seep into these fracture spaces, leading to an increase in $\mathrm{HBL}^{23}$. Vertebral cavity and muscle space also provide storage cavity for HBL.

In our study, the numbers of fractured vertebrae and numbers of fixed vertebral segments were positively related to $\mathrm{HBL}$, as Chen et al. guessed ${ }^{19}$. A previous study proposed that he number of fractured vertebrae was the risk factor of HBL in percutaneous kyphoplasty surgery ${ }^{24}$. Ju et al. held that ALIF was associated with substantial perioperative $\mathrm{HBL}$, and the inclusion of $\mathrm{L} 4 / 5$ in the procedure were significant risk factors for increased blood loss ${ }^{18}$. However, we found that the number of fixed segments was an independent risk factor for hidden blood loss, and the fracture level was not included in our data. We will further explore the relationship between fracture level and hidden blood loss in the future. 
Our previous studies had shown that muscle thickness is also an independent risk factor for hidden blood loss in spinal surgery ${ }^{25}$, thicker muscle may be associated with larger penetrable tissue compartments, allowing blood to ooze into the tissue cavity ${ }^{26}$. Jiang et al. ${ }^{27}$ found that posterior cervical soft tissue was positively correlated with both TBL and HBL in the expansive open-door laminoplasty (EOLP). But the muscle thickness or subcutaneous fat thickness was not clarified as a risk factor in this study. We think that this might be related to the less muscle damage caused by minimally invasive surgery. Therefore, we still need to further study the relationship between muscle thickness and HBL in the setting of spine surgery.

Excessive blood loss can increase the possibility of blood transfusion, which is associated with transfusion reactions, anaphylactic reaction, infections and delayed recovery ${ }^{28}$. Furthermore, excessive blood loss can prolong the hospitalization time and increase the use of medication ${ }^{29}$. The TBL, percentage of vertebral height loss, percentage of vertebral height restoration, numbers of fractured vertebrae, and numbers of fixed vertebral segments should be correctly understood before operation to ensure the safety of patient treatment.

\section{Conclusion}

Consequently, MIPTSF is associated with substantial HBL. More importantly, the total blood loss, percentage of vertebral height loss, percentage of vertebral height restoration, numbers of fractured vertebrae, and numbers of fixed vertebral segments were independent risk factors for HBL. It is important for surgeons to be aware of $\mathrm{HBL}$, to avoid complications related to blood loss. Accurate perioperative HBL assessment can help prevent complications and improve rehabilitation.

\section{Abbreviations}

MIPTSFM: Minimally invasive percutaneous transpedicular screw fixation; PLC: Posterior ligamentous complex; PBV: Patient's blood volume; VBL: Visible blood loss; HBL: Hidden blood loss; TBL: Total blood loss; Hct: Hematocrit; Hb: Hemoglobin; PT: Prothrombin time; APTT: Activated partial thromboplastin time; TT, Thrombin time; PLT, Platelet; MRI, Magnetic resonance imaging; VHL: Vertebral height loss; VHR: Vertebral height restoration; THA: Total hip arthroplasty; TKA: Total knee arthroplasty; ALIF: Anterior lumbar fusion; PLIF: Posterior lumbar fusion.

\section{Declarations}

\section{Acknowledgements}

We would like to thank all the participants in the studies.

\section{Authors' contributions}


YX contributed to the study design, the writing of the paper, and drafting of the manuscript. LZH performed the surgeries and participated in the design of the study. KGM, YM and WTZ collected and analyzed the data. LZH reviewed and edited the manuscript. All authors read and approved the final manuscript.

\section{Funding}

This study was supported by LiaoNing Revitalization Talents Program (XLYC1807131), the Science and Technology Innovation Foundation of Dalian (2020JJ27SN070). The funders had no role in the study design, data collection and analysis, decision to publish, or preparation of the manuscript.

\section{Availability of data and materials}

All data used and analyzed during this study are available from the corresponding author upon reasonable request.

\section{Ethics approval and consent to participate}

This research was approved by the ethics committee of the First Affiliated Hospital of Dalian Medical University. And agreement to participate was given by the participants. Because of the retrospective nature of the study, informed consent was waived.

\section{Consent for publication}

Written informed consent for publication of their clinical details and/or clinical images was obtained from the patient/parent/guardian/relative of the patient.

\section{Competing interests}

The authors declare that they have no competing interests.

\section{References}

1. Tian NF, Wu YS, Zhang XL, et al. Fusion versus nonfusion for surgically treated thoracolumbar burst fractures: a meta-analysis. PloS one 2013;8:e63995.

2. Fernández-de Thomas RJ, De Jesus O. Thoracolumbar Spine Fracture. StatPearls. Treasure Island (FL): StatPearls Publishing Copyright (C) 2020, StatPearls Publishing LLC., 2020.

3. Kim DY, Lee SH, Chung SK, et al. Comparison of multifidus muscle atrophy and trunk extension muscle strength: percutaneous versus open pedicle screw fixation. Spine 2005;30:123-9.

4. Gnanenthiran SR, Adie S, Harris IA. Nonoperative versus operative treatment for thoracolumbar burst fractures without neurologic deficit: a meta-analysis. Clinical orthopaedics and related research 2012;470:567-77. 
5. Wu H, Fu C, Yu W, et al. The options of the three different surgical approaches for the treatment of Denis type A and B thoracolumbar burst fracture. European journal of orthopaedic surgery \& traumatology : orthopedie traumatologie 2014;24:29-35.

6. Wang H, Zhou Y, Li C, et al. Comparison of Open Versus Percutaneous Pedicle Screw Fixation Using the Sextant System in the Treatment of Traumatic Thoracolumbar Fractures. Clinical spine surgery 2017;30:E239-e46.

7. Pannu CD, Farooque $K$, Sharma V, et al. Minimally invasive spine surgeries for treatment of thoracolumbar fractures of spine: A systematic review. Journal of clinical orthopaedics and trauma 2019;10:S147-s55.

8. Ansar MN, Hashmi SM, Colombo F. Minimally Invasive Spine (MIS) Surgery in Traumatic Thoracolumbar Fractures: A Single-Center Experience. Asian journal of neurosurgery 2020;15:76-82.

9. Kaye ID, Passias P. Minimally Invasive Surgery (MIS) Approaches to Thoracolumbar Trauma. Bulletin of the Hospital for Joint Disease (2013) 2018;76:71-9.

10. Ogura Y, Dimar li JR, Gum JL, et al. Hidden blood loss following 2- to 3-level posterior lumbar fusion. The spine journal : official journal of the North American Spine Society 2019;19:2003-6.

11. Sehat KR, Evans R, Newman JH. How much blood is really lost in total knee arthroplasty?. Correct blood loss management should take hidden loss into account. The Knee 2000;7:151-5.

12. Smorgick Y, Baker KC, Bachison CC, et al. Hidden blood loss during posterior spine fusion surgery. The spine journal : official journal of the North American Spine Society 2013;13:877-81.

13. Xu D, Ren Z, Chen X, et al. The further exploration of hidden blood loss in posterior lumbar fusion surgery. Orthopaedics \& traumatology, surgery \& research : OTSR 2017;103:527-30.

14. Nadler SB, Hidalgo JH, Bloch T. Prediction of blood volume in normal human adults. Surgery 1962;51:224-32.

15. Gross JB. Estimating allowable blood loss: corrected for dilution. Anesthesiology 1983;58:277-80.

16. Beghé $C$, Wilson A, Ershler WB. Prevalence and outcomes of anemia in geriatrics: a systematic review of the literature. The American journal of medicine 2004;116 Suppl 7A:3s-10s.

17. Cao D, Zhang S, Yang F, et al. Hidden blood loss and its influencing factors after percutaneous kyphoplasty surgery: A retrospective study. Medicine 2018;97:e0435.

18. Ju H, Hart RA. Hidden blood loss in anterior lumbar interbody fusion (ALIF) surgery. Orthopaedics \& traumatology, surgery \& research : OTSR 2016;102:67-70.

19. Chen ZX, Sun ZM, Jiang C, et al. Comparison of Hidden Blood Loss Between Three Different Surgical Approaches for Treatment of Thoracolumbar Fracture. Journal of investigative surgery : the official journal of the Academy of Surgical Research 2019;32:755-60.

20. Miao K, Ni S, Zhou X, et al. Hidden blood loss and its influential factors after total hip arthroplasty. Journal of orthopaedic surgery and research 2015;10:36.

21. Erskine JG, Fraser C, Simpson R, et al. Blood loss with knee joint replacement. Journal of the Royal College of Surgeons of Edinburgh 1981;26:295-7. 
22. Pattison E, Protheroe K, Pringle RM, et al. Reduction in haemoglobin after knee joint surgery. Annals of the rheumatic diseases 1973;32:582-4.

23. Guglielmino A, Sorbello M, Barbagallo G, et al. Osteoporotic vertebral compression fracture pain (back pain): our experience with balloon kyphoplasty. Minerva anestesiologica 2007;73:77-100.

24. Wu YS, Zhang H, Zheng WH, et al. Hidden blood loss and the influential factors after percutaneous kyphoplasty surgery. European spine journal : official publication of the European Spine Society, the European Spinal Deformity Society, and the European Section of the Cervical Spine Research Society 2017;26:1878-83.

25. Zhou Y, Fu X, Yang M, et al. Hidden blood loss and its possible risk factors in minimally invasive transforaminal lumbar interbody fusion. Journal of orthopaedic surgery and research 2020;15:445.

26. Gao F, Guo W, Sun W, et al. Correlation between the coverage percentage of prosthesis and postoperative hidden blood loss in primary total knee arthroplasty. Chinese medical journal 2014;127:2265-9.

27. Jiang $\mathrm{C}$, Chen TH, Chen ZX, et al. Hidden blood loss and its possible risk factors in cervical open-door laminoplasty. The Journal of international medical research 2019;47:3656-62.

28. Willner D, Spennati V, Stohl S, et al. Spine Surgery and Blood Loss: Systematic Review of Clinical Evidence. Anesthesia and analgesia 2016;123:1307-15.

29. Liang J, Liu H, Huang $X$, et al. Using tranexamic acid soaked absorbable gelatin sponge following complex posterior lumbar spine surgery: A randomized control trial. Clinical neurology and neurosurgery 2016;147:110-4.

\section{Tables}

Table1. Patients demographics. 


\begin{tabular}{|c|c|c|}
\hline Parameters & & Statistics \\
\hline Total patients $(n)$ & & 146 \\
\hline \multirow[t]{2}{*}{$\operatorname{Sex}(n)$} & Male & 106 \\
\hline & Female & 40 \\
\hline Age, yr & & $42.31 \pm 7.90$ \\
\hline $\mathrm{BMI}, \mathrm{kg} / \mathrm{m} 2$ & & $25.33 \pm 3.13$ \\
\hline Muscle thickness, mm & & $31.61 \pm 7.84$ \\
\hline Subcutaneous fat thickness, $\mathrm{mm}$ & & $19.84 \pm 6.19$ \\
\hline Muscle thickness/Subcutaneous fat thickness & & $1.64 \pm 0.26$ \\
\hline Smoking (n) & & 35 \\
\hline Drinking $(\mathrm{n})$ & & 17 \\
\hline Diabetes mellitus ( $\mathrm{n}$ ) & & 10 \\
\hline Hypertension (n) & & 13 \\
\hline Low immunity(n) & & 3 \\
\hline Using hormones(n) & & 8 \\
\hline Combining with other fractures(n) & & 20 \\
\hline \multirow[t]{3}{*}{ Fracture classification(n) } & A1 & 87 \\
\hline & $\mathrm{A} 2$ & 23 \\
\hline & A3 & 36 \\
\hline Preoperative HCT,\% & & $38.23 \pm 4.41$ \\
\hline Postoperative HCT,\% & & $35.18 \pm 4.51$ \\
\hline PBV, L & & $4.87 \pm 0.71$ \\
\hline TBL, ml & & $403.45 \pm 182.25$ \\
\hline VBL, ml & & $239.45 \pm 130.17$ \\
\hline VHL, \% & & $45.58 \pm 11.08$ \\
\hline VHR, \% & & $23.43 \pm 9.47$ \\
\hline Numbers of fractured vertebrae & & $1.18 \pm 0.45$ \\
\hline Numbers of fixed vertebral segments & & $3.03 \pm 0.66$ \\
\hline Hospital stay, d & & $12.28 \pm 2.64$ \\
\hline
\end{tabular}




\begin{tabular}{|ll|}
\hline Surgical duration, min & $120.14 \pm 34.06$ \\
\hline Preoperative $\mathrm{Hb}, \mathrm{g} / \mathrm{I}$ & $124.86 \pm 14.36$ \\
\hline Postoperative $\mathrm{Hb}, \mathrm{g} / \mathrm{I}$ & $103.92 \pm 13.67$ \\
\hline APTT, s & $11.46 \pm 1.22$ \\
\hline TT, s & $32.90 \pm 6.18$ \\
\hline Fibrinogen, g/l & $17.65 \pm 1.25$ \\
\hline PLT, $109 / \mathrm{I}$ & $3.37 \pm 0.80$ \\
\hline
\end{tabular}

BMI, Body mass index; HCT, Hematocrit; PBV, Patient's blood volume; TBL, Total blood loss; VBL, Visible blood loss; VHL, Vertebral height loss; VHR, Vertebral height restoration; HB, Hemoglobin; PT, Prothrombin time; APTT, Activated partial thromboplastin time; TT, Thrombin time; PLT, Platelet.

Table2. Changes in HCT and HB level following MIPTSF.

\begin{tabular}{|lllll|}
\hline Parameters & Mean & SD & t & P \\
\hline Preoperative and postoperative HCT, \% & 3.0486 & 1.3208 & 27.890 & .000 \\
\hline Preoperative and postoperative HB囚g/L & 20.945 & 11.672 & 21.683 & .000 \\
\hline
\end{tabular}

MIPTSF, Minimally invasive percutaneous transpedicular screw fixation; HCT, Hematocrit; HB, Hemoglobin; SD, Standard deviation.

Table 3 Results of the Pearson or Spearman correlation analysis for HBL. 


\begin{tabular}{|c|c|c|}
\hline Parameters & Sig & $\mathbf{P}$ \\
\hline Gender & -.102 & .219 \\
\hline Age & -.051 & .539 \\
\hline BMI & .455 & .000 \\
\hline Muscle thickness & .778 & .000 \\
\hline Subcutaneous fat thickness & .646 & .000 \\
\hline Muscle thickness/Subcutaneous fat thickness & -.106 & .204 \\
\hline Smoking & .036 & .670 \\
\hline Drinking & .001 & .993 \\
\hline Diabetes mellitus & -.164 & .048 \\
\hline Hypertension & -.077 & .357 \\
\hline Low immunity & -.037 & .655 \\
\hline Using hormones & -.043 & .608 \\
\hline Combining with other fractures & -.145 & .081 \\
\hline Fracture classification & .519 & .000 \\
\hline PBV & .016 & .852 \\
\hline TBL & .706 & .000 \\
\hline VBL & .128 & .125 \\
\hline VHL & .938 & .000 \\
\hline VHR & .921 & .000 \\
\hline Numbers of fractured vertebrae & .625 & .000 \\
\hline Numbers of fixed vertebral segments & .746 & .000 \\
\hline Hospital stay & -.061 & .466 \\
\hline Surgical duration & .356 & .000 \\
\hline PT & -.323 & .000 \\
\hline APTT & .590 & .000 \\
\hline TT & .172 & .038 \\
\hline Fibrinogen & -.040 & .629 \\
\hline PLT & -.074 & .375 \\
\hline
\end{tabular}


HBL, Hidden blood loss; BMI, Body mass index; PBV, Patient's blood volume; TBL, Total blood loss; VBL, Visible blood loss; VHL, Vertebral height loss; VHR, Vertebral height restoration; PT, Prothrombin time; APTT, Activated partial thromboplastin time; TT, Thrombin time; PLT, Platelet.

Table 4 : Results of multivariate linear regression for HBL.

\begin{tabular}{|c|c|c|c|c|c|}
\hline \multirow[t]{2}{*}{ Parameters } & \multicolumn{2}{|c|}{ Unstandardized } & \multirow{2}{*}{$\begin{array}{l}\text { Standardized } \\
\text { B }\end{array}$} & \multirow[t]{2}{*}{$t$} & \multirow[t]{2}{*}{$\mathbf{P}$} \\
\hline & $\beta$ & SE & & & \\
\hline Constant & -238.468 & 48.114 & & -4.956 & .000 \\
\hline TBL & .085 & .018 & .139 & 4.735 & .000 \\
\hline VHL & 4.353 & .582 & .431 & 7.479 & .000 \\
\hline VHR & 3.823 & .669 & .323 & 5.713 & .000 \\
\hline Numbers of fractured vertebrae & 20.057 & 7.954 & .081 & 2.522 & .013 \\
\hline Numbers of fixed vertebral segments & 19.468 & 6.184 & .114 & 3.148 & .002 \\
\hline BMI & .352 & .925 & .010 & .380 & .704 \\
\hline Muscle thickness & .859 & .866 & .060 & .992 & .323 \\
\hline Subcutaneous fat thickness & -1.184 & .893 & -.065 & -1.326 & .187 \\
\hline Surgical duration & -.134 & .085 & -.041 & -1.578 & .117 \\
\hline PT & -.632 & 2.184 & -.007 & -.289 & .773 \\
\hline APTT & -.704 & .534 & -.039 & -1.318 & .190 \\
\hline TT & 1.665 & 1.980 & .019 & .841 & .402 \\
\hline Fracture classification (A2) & -5.512 & 7.073 & -.018 & -.779 & .437 \\
\hline Fracture classification (A3) & 10.699 & 8.057 & .041 & 1.328 & .187 \\
\hline
\end{tabular}

HBL, Hidden blood loss; TBL, Total blood loss; VHL, Vertebral height loss; VHR, Vertebral height restoration; BMI, Body mass index; PT, Prothrombin time; APTT, Activated partial thromboplastin time; TT, Thrombin time.

\section{Figures}




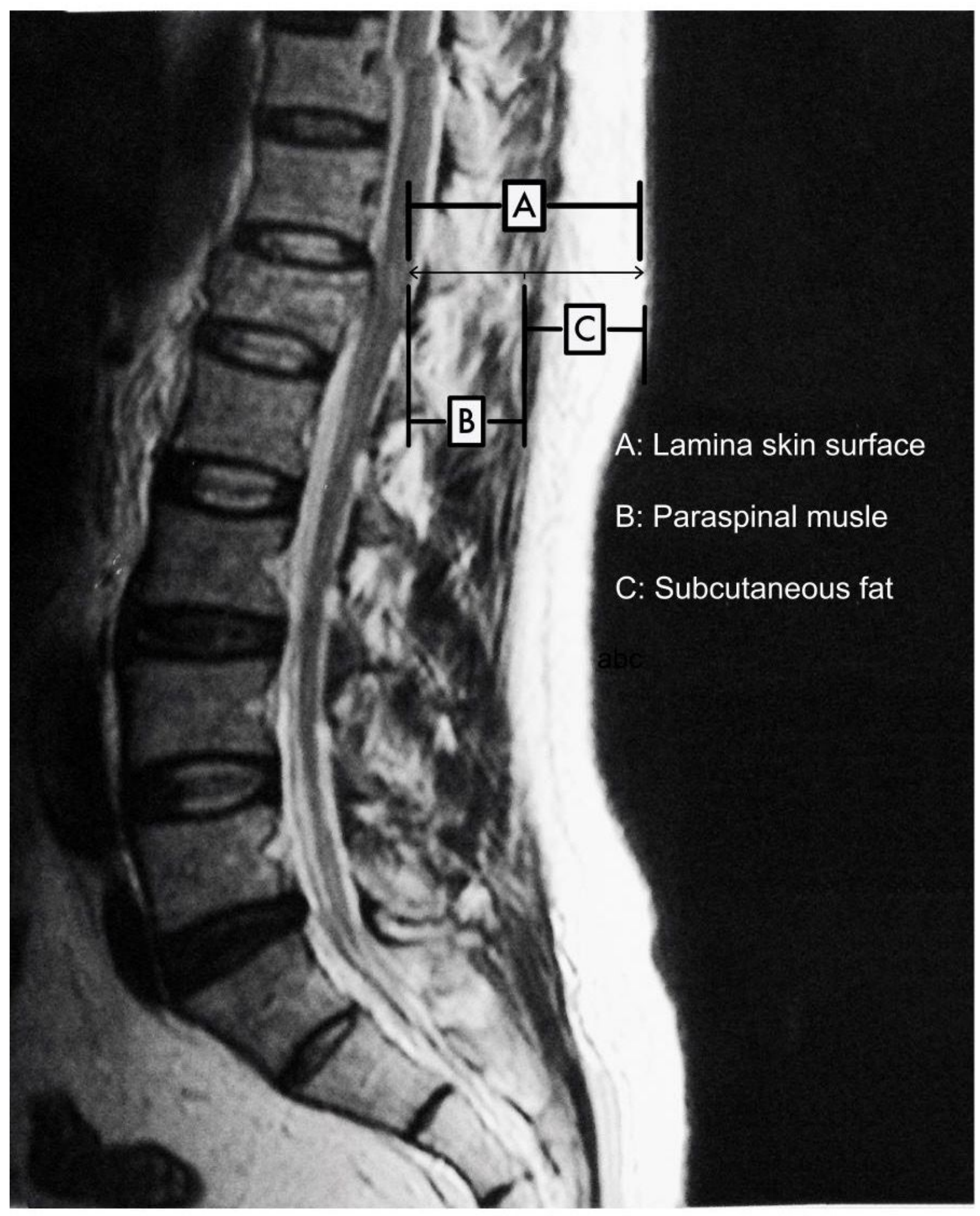

\section{Figure 1}

Diagram of the method used to measure thickness of the paraspinal muscles, subcutaneous fat, and lamina at the skin surface at the level of L1 using sagittal views was determined on T2-weighted MRI. 


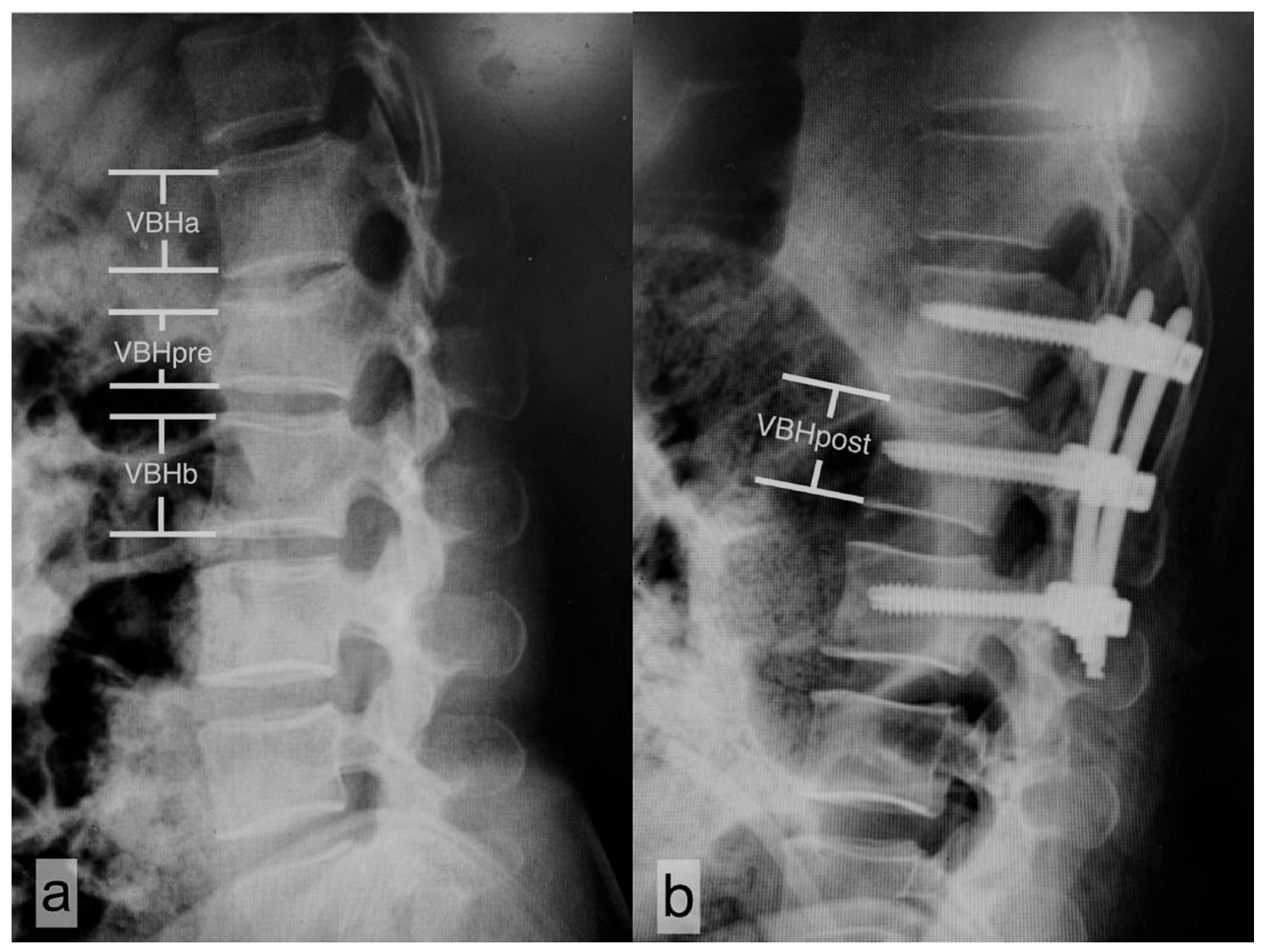

Figure 2

Diagram of the method for measuring the percentages of vertebral height loss $(\mathrm{VHL})$ and vertebral height restoration (VHR) on sagittal plain radiograph. (a) Preoperative, (b) Postoperative

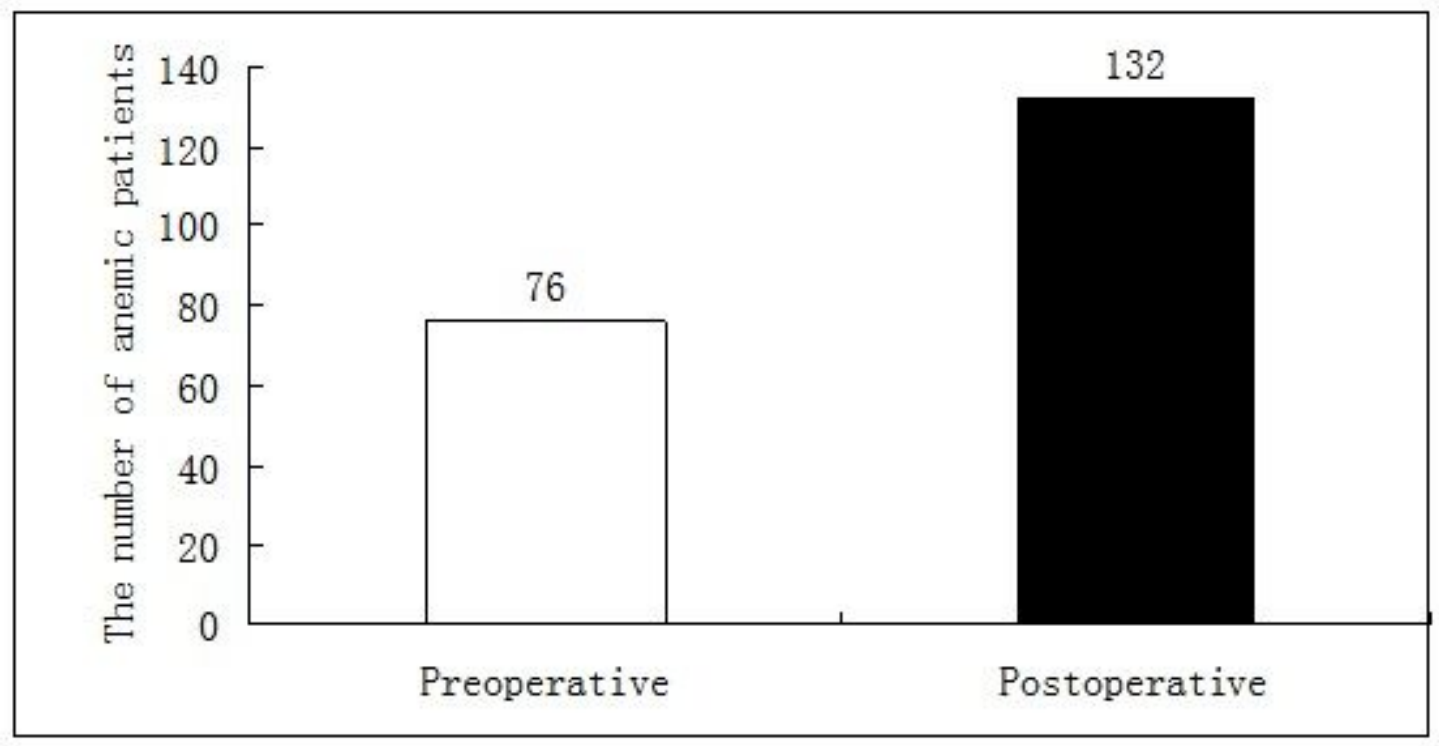


Figure 3

The number of anemic patients. 